\title{
Generalizing Automatically Generated Selectional Patterns
}

\author{
Ralph Grishman and John Sterling \\ Computer Science Department, New York University \\ 715 Broadway, 7th Floor, New York, NY 10003, U.S.A. \\ \{grishman,sterling\}@oles.nyu.cdu
}

\begin{abstract}
Frequency information on co-occurrence patterns can be automatically collected from a syntactically analyzed corpus; this information can then serve as the basis for selectional constraints when analyzing new text from the same domain. This information, however, is necessarily incomplete. We report on measurements of the degree of selectional coverage obtained with different sizes of corpora. We then describe a technique for using the corpus to identify selectionally similar terms, and for using this similarity to broaden the selectional coverage for a fixed corpus size.
\end{abstract}

\section{Introduction}

Selectional constraints specify what combinations of words are acceptable or meaningful in particular syntactic relations, such as subjoct-verb-object or headmodifier relations. Such constraints are necessary for the accurate analysis of natural language text. Accordingly, the acquisition of these constraints is an cssential yet time-consuming part of porting a natural language system to a new domain. Several research groups have attempted to automate this process by collecting co-occurrence patterns (e.g., subject-verb-object patterns) from a large training corpus. 'These patterns are then used as the source of selectional constraints in analyzing new text.

'The initial succosses of this approach raise the question of how large a training corpus is required. Any answer to this question must of course be relative to the degree of coverage required; the set of selectional patterns will never be $100 \%$ complete, so a large corpus will always provide greater coverage. We attempt to shed to some light on this question by processing a large corpus of text from a broad clomain (business news) and observing how selectional coverage increases with domain size.

In many cases, there are limits on the anount of training text available. We therefore also consider how coverage can be increased using a fixed amount of text. The most straightforward acquisition procedures build selectional patterns containing only the specific word combinations found in the training corpus, Cireater coverage can be obtained by genoralizing from the patterns collected so that patterns with semantically related words will also be considered acceptable. In most cases this has been dono using manually-created word classes, generalizing from specific words to their classes $[12,1,10]$. If a pre-existing set of classes is used (as in [10]), there is a risk that the classes available may not match the nceds of the task. If classes are created specifically to capture selectional constraints, there may be a substantial manual burden in moving to a new domain, since at least some of the semantic word classes will be domain-specific.

We wish to avoid this manual component by automatically identifying semantically related words. This can be done using the co-occurrence data, i.e., by identifying words which occur in the same contexts (for example, verbs which occur with the same subjects and objects). From the co-occurrence data one can conpute a similarity relation between words $[8,7]$. 'This similarity information can then be used in several ways. One approach is to form word chusters based on this similarity relation [8]. This approach was taken by Sekine et al. at UMIST, who then used these clusters to generalize the semantic pattorns [11]. Pereira et al. [9] used a variant of this approach, "soft clusters", in which words can be members of different clusters to difforent degrees.

An alternative approach is to use the word similarity information directly, to infer information about the likelihood of a co-occurrence pattern from information about patterns involving similar words. This is the approach we have adopted for our current experimonts [6], and which has also been employed by Dagan et al. [2]. We compute from the co-occurrence data a "confusion matrix", which measures the interchangeability of words in particular contexts. Wo then use the confusion matrix directly to generalize the semanttic patterns.

\section{Acquiring Semantic Patterns}

Based on a series of experiments over the past tiwo years $[5,6]$ we have developed the following procedure 
for accuiring semantic pattems from a text compus:

1. Parse the training corpus using a broad-coverage grammar, and regularize the parses to produce something akin to an IFG f-structure, with explic it.ly labeled syntactic relations such as SU B.J liO'T' and obstsc,

2. Lixtract from the regularized parse a series of triples of the form

head syntactic-relation hoad-of-argunent
/modifier

We will use the notation $\left\langle w_{i} r w_{j}\right\rangle$ for such a triple, and $\left\langle r w_{j}\right\rangle$ lor a relation argument pair.

3. Computo the frequency fr of eath head and oach triple in the corpus. If a sentence produces $\mathrm{N}$ parses, a triple generated from a single parso has weight $1 / N$ in the totial.

We will use the notalion $F^{\prime}\left(\left\langle u_{i} r w_{j}\right\rangle\right)$ for the fre. quency of a triple, and thend $\left(w_{i}\right)$ for the frequency with which $w_{i}$ appears as a head in a parse tree."

For example, the sentence

Mary likes young linguists from limerick.

would produce the regularized syntactic structure

(s like (subject (np) Mary))

(object, (np, linguist (a-pos young)

(from (up limericks)) ))

from which the following four triples are generated:

$\begin{array}{lll}\text { like } & \text { subject } & \text { Mary } \\ \text { like } & \text { object } & \text { linguist } \\ \text { linguist } & \text { a-pos } & \text { young } \\ \text { linguist } & \text { from } & \text { limerick }\end{array}$

Given the frequency information $F^{\prime}$, we can then estimate the probability that a particular head $w_{i}$ appears with a particulas argument or modilies $\left\langle r u_{j}\right\rangle$ :

$$
\frac{l^{\prime}\left(<w_{i} v^{\prime} w_{j}>\right)}{l_{\text {head }}^{\prime}\left(w_{i}\right)}
$$

'This probability information would then be used in scoring alternative parse trees. for the evaluation below, however, we will ase the frequency daba $l^{\prime}$ directly.

Step 3 (the triples extraction) includes a number of special cases:

\footnotetext{
${ }^{1}$ Bub with somewhat more regularization than is done in If If $i$; in particular, passive structures are converted to corresponding active forms.

${ }^{2} \mathrm{Note}$

thith

l'herd $\left(w_{i}\right)$

diflerent from $W\left(w_{i}\right.$ appears as a head in a triple) since a single liead in a parse trec may produce several sich triples, one for each argument or modifier of that hear.
}

(a) if a verb has a separable particle (e.g., "out" in "carry out"), this is attached to the head (to cre. ate the head carry-oul) and not treated as a separate relation. Diflerent particles often correspond to very different senses of a verb, so this avoids conflating the subject and object distributions of these different senses.

(b) il the verh is "be", we generatio a relation becomplement between the sulject and the predicate. complenent.

(c) triples in which cither the head or the argument is a pronoun are discarded

(d) triples in which the argument is a suburdinate clause are discarded (this inchudes subordinate conjunctions and verbs taking clausal argurnents)

(c) triples indicating negation (with an argunent, of "not" or "never") are ignored

\section{Generalizing Semantic Pat- terns}

The procedure described above produces as set of the quencies and probahility estimates hased on specilic words. "The "traditional" approach to generalizing this; intormation has been to asisign the words to a set of semantic classes, and then to collect the frecunency information on combinations of semantic classes $[12,1]$.

Since at least some of these classes will be domain specific, there las been interest in antomating the acquisition of these classes ats well. This ean be done by chustering together words which appear in the sanue context. Stateting from the file of triples, this involves:

1. collecting for each word the frecuency with which it oceurs in each possible context; for exarnple, for a noun we would colled the frequency with which it occurs as the subject and the object of each verh

2. defining a similarity measure between words, which reflects the mumber of common contexts in which they appear

3. forming chusters based on this similarity measure

Such a procedure was performed by Sokine et al. at UMIS'I' [1]]; these clusters were then manually re viewed and the resulting clusters were used to generalize selectional patiterns. A similar approach to word cluster formation was described by flirschman et al. in 1975 [8]. More recently, J'ercira et al. [9] have described a word clustering nethod using "soft elusters", in which a word can bolong to snveral clusters, with diflerent cluster menbership probabilities.

Cluster creation has the advantiage that the clusters are annenable to mannal review and correction. On the other hand, our experience indieates that suecessful cluster generation depends on rather delicate adjustment of the chustering criteria. We have therefore 
elected to try an approach which directly uses a form of similarity measure to smooth (generalize) the prob. abilities.

Co-occurrence smoothing is a mothod which has been recently proposed for smoothing $n$-gram models [3]. ${ }^{3}$ The core of this method involves the computation of a co-occurrence matrix (a matrix of confusion probabilities) $P_{C}\left(w_{j} \mid u_{i}\right)$, which indicates the probability of word $w_{j}$ occurring in contexts in which word $w_{i}$ occurs, averaged over these contexts.

$$
\begin{aligned}
P_{C}\left(w_{j} \mid w_{i}\right) & =\sum_{s} P\left(w_{j} \mid s\right) P\left(s \mid w_{i}\right) \\
& =\frac{\sum_{s} P\left(w_{j} \mid s\right) P\left(w_{i} \mid s\right) P^{\prime}(s)}{P\left(w_{i}\right)}
\end{aligned}
$$

where the sum is over the set of all possible contexts $s$. In applying this technique to the triples we have collected, we have initially chosen to goneralize (smooth over) the first element of triple. Thus, in triples of the form wordl relation word 2 we focus on word1, treating relation and word: as the context:

$$
\begin{aligned}
& P_{C}\left(w_{i} \mid w_{i}^{\prime}\right) \\
& =\sum_{r, w_{j}} P\left(w_{i} \mid<r w_{j}>\right) \cdot P\left(<r w_{j}>\mid w_{i}^{\prime}\right) \\
& =\sum_{r, w_{j}} \frac{\left.F\left(<w_{i} r w_{j}\right\rangle\right)}{\left.F\left(<r w_{j}\right\rangle\right)} \cdot \frac{\left.F\left(<w_{i}^{\prime} r w_{j}\right\rangle\right)}{\Gamma_{\text {head }}\left(w_{i}^{\prime}\right)} .
\end{aligned}
$$

Informally, we can say that, a large value of $P_{C}\left(w_{i} \mid w_{i}^{\prime}\right)$ indicates that $w_{i}$ is selectionally (semantically) acceptable in the syntactic contexts where word $w_{i}^{\prime}$ appears. For example, looking at the verb "convict", we see that the largest values of $P_{C}($ convict, $x)$ are for $x=$ "acquit" and $x=$ "indict", indicating that "convict" is selectionally acceptable in contexts where words "acquit" or "indict" appear (see Figure 4 for a larger example).

How do we use this information to generalize the triples obtained from the corpus? Suppose we are interested in determining the acceptability of the pattern convict-object-owner, even though this triple does not appear in our training corpus. Since "convict" can appcar in contexts in which "acquit" or "indict" appear, and the patterns acquit-object-owner and indictobject-owner appear in the corpus, we can conclude that the pattern convict-object-owner is acceptable too. More formally, we compute a smoothed triples frequency $F_{S}$ from the observed frequency $F$ by averaging over all words $w_{i}^{\prime}$, incorporating frequency information for $w_{i}^{\prime}$ to the extent that its contexts are also suitable contexts for $w_{i}$ :

$\left.F_{S}\left(<w_{i} r w_{j}\right\rangle\right)=\sum_{w_{i}^{\prime}} P_{C}\left(w_{i} \mid w_{i}^{\prime}\right) \cdot F\left(<w_{i}^{\prime} r w_{j}>\right)$

In order to avoid the gencration of confusion table entrics from a single shared context (which quite often

\footnotetext{
${ }^{3}$ We wish to thank Richard Schwartz of BBN for referring us to this method and article.
}

is the result of an incorrect parse), we apply a filter in generating $I_{C}$ : for $i \neq j$, we generate a non-zero $P_{C}\left(w_{j} \mid w_{i}\right)$ only if the $w_{i}$ and $w_{j}$ appear in at least two common contexts, and there is some common context in which both words occur at least twice. Furthermore, if the value computed by the formula for $P_{C}$ is less than some threshold $\tau_{C}$, the value is laken to be zero; wo have used $\tau_{c}=0.001$ in the experiments reported below. (These filters are not applied for the case $i=j$; the diagonal clements of the confusion matrix are always computed exactly.) Because these filters may yeild an un-normalized confusion matrix (i.e., $\sum_{w_{j}} P_{C}\left(w_{j} \mid w_{i}\right)<1$ ), we renomalize the matrix so that $\sum_{w_{j}} P_{C}\left(w_{j} \mid w_{i}\right)=1$.

A similar approach to pattern generalization, using a similarity measure derived from co-occurrence data, has beon recently described by Dagan et al. [2]. Their approach differs from the one described here in two significant regards: their co-occurrence data is based on linear distance within the sontence, rather than on syntactic relations, and they use a different similarity measure, based on mutual information. 'T'he relative merits of the two similarity measures may need to be resolved empirically; however, we believe that there is a virtue to our non-symmetric measure, because substitutibility in selectional contexts is not a symmetric relation. ${ }^{*}$

\section{Evaluation}

\subsection{Evaluation Metric}

We have proviously [5] described two methods for the evaluation of semantic constraints. For the current cxperiments, we have used one of these methods, where the constraints are evaluated against a set of manually classified semantic triples. ${ }^{5}$

fior this evaluation, we select a small test corpus separate from the training corpus. We parse the corpus, regularize the parses, and cxtract triples just as we did for the semantic acquisition phase. We then manually classify each triple as valid or invalid, depending on whether or not it arises from the correct parse for the sentence. ${ }^{6}$

Wo then establish a threshold $T$ for the weighted triples counts in our training set, and define

\footnotetext{
${ }^{4}$ If $w_{1}$ allows a brouder range of arguments than $w_{2}$, then we can replace $w_{2}$ by $w_{1}$, but not vice versa. For example, we can replace "speak" (which takes a human subject) by "sleep" (which takes an animate subjoct), and still have a selectionally valid pattern, but not the other way around.

"This is similar to tests conducted by Percima et al. [9] and Dagan et al. [2]. 'T'he cited lests, however, were based on selected words or word pairs of high frequency, whereas our test sets involve a representative set of high and low frequency triples.

${ }^{6}$. $\mathrm{Th}$ is is a different criterion from the one used in our earlier papers. In our earlier work, we marked a triple as valid if it could be valid in some sentence in the clomain. We found that it was very difficult to apply such a standard consistently, and have therefore changed to a criterion based on an individual sentence.
} 


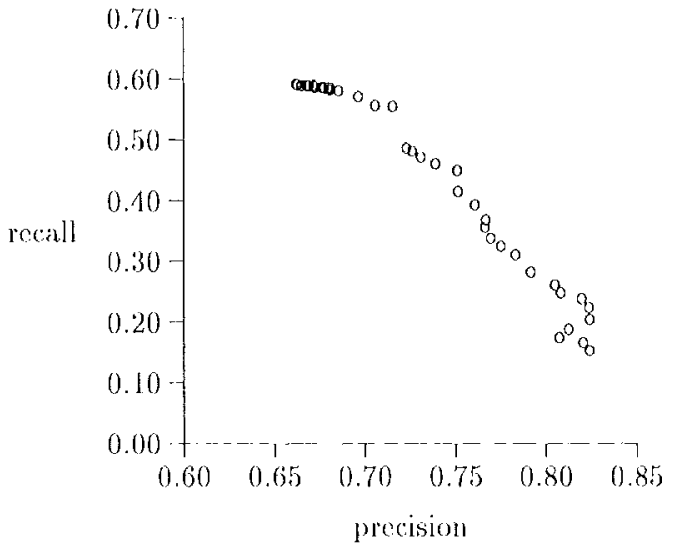

Fignre 1: Recall/precision trade-off using cutire corpuss.

$v_{+}$number of triples in test set which were classified as valid and which appeared in training set with count $>$ '

$v_{\text {- }}$ number of triples in test set which were classified as valid and which appoared in training set with count $\leq T$ '

$i_{+}$enumber of triples in thest set which were classified as invalid and which appeared in training set, with count $>$ ' $T$ '

and then define

$$
\begin{aligned}
\text { recall } & =\frac{v_{+}}{v_{+}+v_{-}} \\
\text {precision } & =\frac{v_{+}}{v_{+}+i_{+}} .
\end{aligned}
$$

By varying the threshold, we can sclect different trade-ofls of recall and precision (at high threshold, we select only a small number of triples which appeared frecuently and in which we therefore have high confidence, thus obtaining a high precision but low recall; conversely, at a low threshold we adruit a much larger number of triples, obtaining a high recall but lower precision).

\subsection{Test Data}

'The training and test corpora were taken from the Wall Street fourmal. In order to get higher-quality parses of these sentences, we disabled some of the recovery mechanisms normally used in our parser. Of the 57,366 sentences in on training corpus, we obtained conplete parses for 34,414 and parses of initial substrings for an additional 12,141 sentences. These parses were then regularized and reluced to triples. We generated a total of 279,233 distinct triples from the corpus.

The test corpus used to generate the triples which were manually classified consisted of 10 articles, also

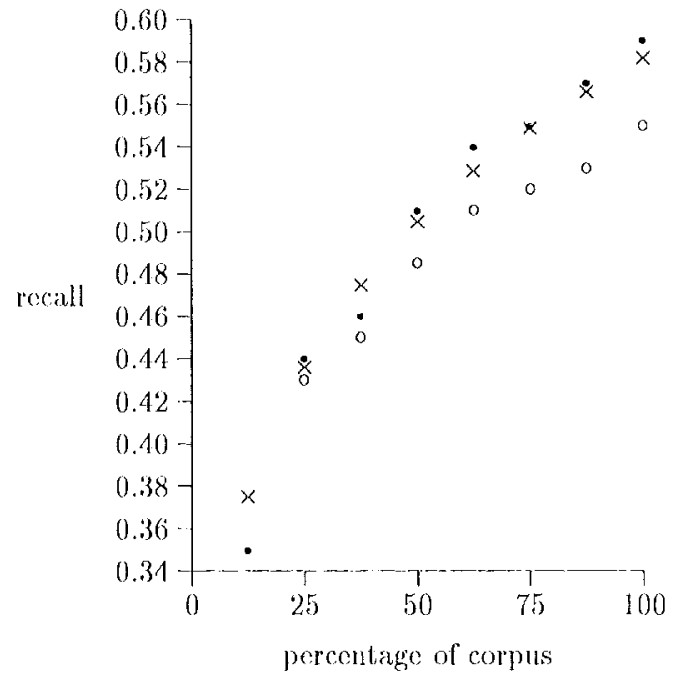

Figure 2: (Xrowth of recall as a function of corpus size (percentage of total corpus used). o = at $72 \%$ precision; - = maximum recall, regardless of precision; $x=$ predicted values for maxirmum recall

from the Wall Street, Journal, distinct, from those in the training set. These articles produced a test set containing a tolal of 1932 triples, of which 1107 were valid and 825 were invalid.

\section{$4.3 \quad$ Results}

\subsubsection{Growth with Corpus Size}

We began by generaling triples from the entire corpus and evaluating the selectional patterns as described above; the resulting recall/precision curve grenerated by varying the threshold is shown in Figure 1.

'To see how pattern coverage improves with corpus size, we divided our training corpus into 8 segments and computed sets of triples based on the first segment, the lirst two segnents, ete. We show in Figure 2 a plot of recall vs. corpus size, both at a constant precision of $72 \%$ and for maximum recall regardless of precision. ${ }^{7}$

The rate of growth of the maximum recall can be understood in terms of the frequency distribution of triples. In our carlier work [4] we fit the growth dala to enrves of the form $1-\exp (-\beta x)$, on the assumption that all selectional patterns are equally likely. 'This may have been a roughly accurate assmmption for that application, involving semantic-class based patterns (rather than word-based patterns), and a rather sharply circumscribed sublanguage (medical reports). for the (word-level) patterns described here, however, the distribution is quite skewed, with a small number of very-high-frequency pattems, which results in dif-

\footnotetext{
${ }^{7} \mathrm{No}$ data point is shown for $72 \%$ precision for the first seffment alone because we are not able to reach a precision of $72 \%$ with a single seginent.

"The number of high-frequency patterns is accentuated by
} 


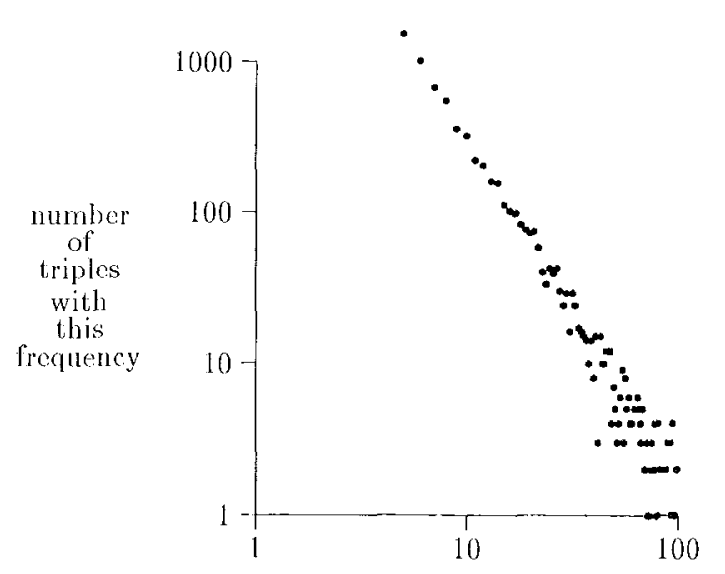

frequency of triple in training corpus

Figure 3: Distribution of frequencies of triples in training corpus. Vertical scale shows number of triples with a given frequency.

ferent growth curves. Figure 3 plots the number of distinct triples per unit frequency, as a function of frequency, for the entire training corpus. This data can be very closely approxinated by a function of the form $N\left(r^{\prime}\right)=a F^{\prime-\alpha}$, where $x=2.2 .^{9}$

To derive a growth curve for maximum recall, we will assume that the frequency distribution for triples selected at random follows the same forn. Let $p(T)$ represent the probability that a triple chosen at random is a particular triple 7 . I,et $P(p)$ be the density of triples with a given probability; i.e., the number of triples with probabilitics betwen $p$ and $p+c$ is $\epsilon P(p)$ (for small $\epsilon$ ). Then we are assuming that $P(p)=\kappa p^{-\alpha x}$, for $p$ ranging from some mininum probability $p_{m i n}$ to L. For a triple $T$, the probability that wo would find at. least one instance of it in a corpus of $\tau$ triples is approximately $\mathrm{I}-e^{-\tau p(T)}$. The maximum recall for a corpus of $\tau$ triples is the probability of a given triple (the "test triple") being selected at random, multiplied by the probability that that triple was found in the training corpus, summed over all triples:

$$
\sum_{T} p(T) \cdot\left(1-e^{-\tau p(T)}\right)
$$

which can be computed using the density function

$$
\begin{aligned}
& \int_{p_{\min }}^{1} p \cdot P(p) \cdot\left(1-c^{-r p}\right) d p \\
= & \int_{p_{m i n}}^{1} \kappa p \cdot p^{-\alpha v}\left(1-c^{-\tau p}\right) d p
\end{aligned}
$$

By selecting an appropriate value of $\kappa$ (and corresponding $p_{\min }$ so that the total probability is 1 ), we can get a

the fact that our lexical scammer replaces all identifiable company names by the token a-company, all currency values by acurrency, etc. Many of the highest frequency triples involve such tokens.

'This is quite similar to a Zipf's law distribution, for which $x=2$.

\begin{tabular}{|l|r|}
\hline$w$ & $P_{C}($ bond $\mid w)$ \\
\hline eurobond & 0.133 \\
foray & 0.128 \\
objective & 0.093 \\
marriage & 0.089 \\
note & 0.071 \\
maturity & 0.068 \\
subsidy & 0.057 \\
veteran & 0.046 \\
commitinent & 0.046 \\
debenture & 0.046 \\
activism & 0.044 \\
mile & 0.043 \\
coupon & 0.038 \\
security & 0.038 \\
yicld & 0.037 \\
issuc & 0.036 \\
\hline
\end{tabular}

Figure 4: Nouns closely related to the noun "bond", ranked by $P_{C}$.

good matcl to the actual maximum recall values; these computed values are shown as $x$ in Figure 2 . Except for the smallest data set, the agreement is quite good considering the very simple assumptions made.

\subsubsection{Smoothing}

In order to increase our coverage (recall), we then applied the smoothing procedure to the triples from our training corpus. In testing our procedure, we first generated the confusion matrix $P_{C}$ and cxamined some of the entries. Figure 4 shows the largest cntries in $P_{C}$ for the noum "bond", a common word in the Wall Street Journal. It is clear that (with some odd exceptions) most of the words with high $P_{C}$ : values are semantically related to the original word.

'To evaluate the effectiveness of our smoothing procedure, we have plotted recall vs. precision graphs for both unsmoothed and smoothed frequency data. 'The results are shown in Figure 5. Over the range of precisions where the two curves overlap, the smoothed data performs beticr at low precision/high recall, whereas the unsmoothed data is better at high precision/low recall. In addition, smoothing substantially extends the level of recall which can be achicved for a given corpus size, although at some sacrifice in precision.

Intuitively we can understand why these curves should cross as they do. Smoothing introduces a ccrtain degree of additional error. As is evident from rigure 4 , some of the confusion matrix entries are spurious, arising from such sources as incorrect parses and the conflation of word senses. In addition, some of the triples being generalized are themselves incorrect (note that even at high threshold the precision is below $90 \%$ ). The net result is that a portion (roughly $1 / 3$ to $1 / 5$ ) of 


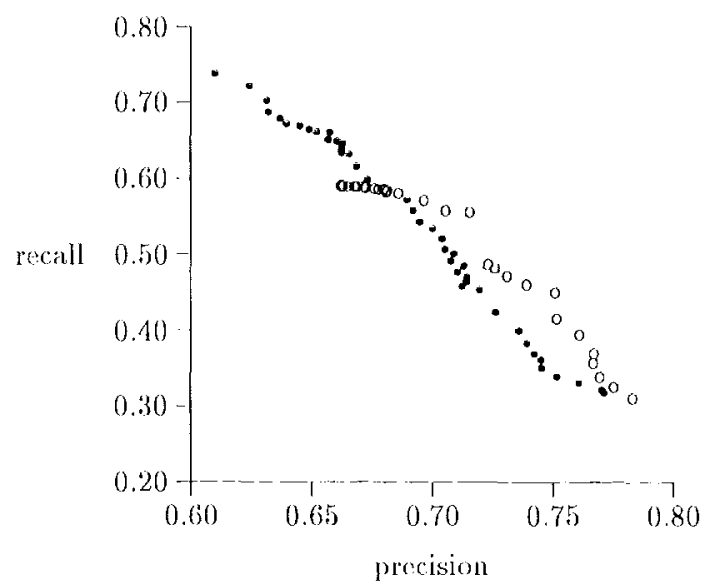

Figure 5: Benefits of smoothing for largest corpus: o $=$ unsmoothed data, $\bullet=$ smoolhed data.

the triples added by smoothing are incorrect. At low levels of precision, this produces a net gain on the precision/recall curve; at higher levels of precision, there is a net loss. In any event, smoothing cloes allow for substantially higher levels of recall than are possible. without smoathing.

\section{Conclusion}

We lave denonstrated how selectional patterns can be automatically accuared from a corpus, and how solectional coverage gradually increases with the size of the training corpus. We have also demonstrated that -.. for a given corpus size coverage can be significantly improved by using the corpus to identify selectionally related terns, and using these similaritios to generalize? the patterns observed in the training corpus. 'L'his is consistent with other recent results using related technicues $[2,9]$.

We believe that these techniques can be further improved in several ways. The experinents reported above have only generalized over the lirst, (head) position of the triples; we need to measure the eflect of generalizing over the argument position as well. With larger corpora it may also be teasible to use larger patterus, inchuding in particular subject-verb-object pat. terns, and thus reduce the confusion dhe to treating different words sensises as common contiexts.

\section{References}

[1] Jing-Shin Chang, Vih-ten I.uo, and Koht-Yih Su. (XPSM: A generalized probabilistic semantic model for ambiguity resolution. In Procecdings of the 30th Annual Mecting of the Assn. for Computational linguistics, pages 177-184, Newark, DE, June 1992.
[2] Ido Dagan, Shaul Marcus, and Shaul Markovilch. Contextual word similarity and estimation from sparse data. In Procedings of the 31 st Annual Mecting of the Assn. for Computational linguistics, pages 31 37, Columbus, OH, Jume 1993.

[3] U. lissen and V. Steinbiss. Cooccurrence. stmoothing for stochastic language modeling. In ICASSPg, pages 1 161-1 164, San lirancisco, CA, May 1992.

[4] R. Grishman, I. Mirschman, and N.'I'. Nhan. Dis covery procedures for sublanguage selectional pattorns: Initial experiments. Compulalional Linguisties, 12(3):205 16, 1986.

[5] Ralph Grishman and John Sterling. Acquisition of sclectional patiterns. In l'roce lith $\mathrm{lnt}$ 'l Conf. Computational Linguisties (COLING ges), Nantes, f'rance, July 1992.

[6] Ralph ('rishnan and John Sterling. Smoothing of automatically generated selectional constraints. In P'rocedings of the Human Language 'T'chnology Workshop, Princolon, NJ, March 1993.

[7] Donald Hindle Nonn classification from predicate-argument structures. In Procecdings of the s8th Annual Mecling of the Assu. for Computational linguisties, pages $268-275$, June 1990.

[8] Jiynetle Ilirschman, Ratph Grishman, and Naomi Sager. (irammatically-based automatic word class formation. Information Processing and Managemenl, $11(1 / 2): 39-57,1975$.

[9] l'ernando P'ereira, Nattali T'ishby, and Lillian Lee. Distributional clustering of Fnglish words. In Procedings of the 3Ist Annual Mceting of the Assn. for Compulational linguistics, pages $18: 3$ 190, Columbus, OH, June 1993.

[10] Philip Resnik. A class-based approach to lexi. cal cliscovery. In Procectings of the 30th Annual Mecting of the Assn. for Compulational Linguisties, Newark, 1)ti, June 1992.

[11] Satoshi Sekine, Sofia Anamiadou, Jeremy Carroll, and Jun'jchi 'T'sujii. Linguistic kuowledge gentcrator. In l'roc. 1/4h Inl'l Conf. Compulational linguistess (COLIN(790), pages 5600566 , Nantes, Finace, fuly 1992.

[12] Paola Velardi, Maria 'l'eresa Pamienza, and Michela tiasolo. How to encode semantic knowlodge: A method for moaning representation and computer-aided acquisition. Compulational hinguistics, $17(2): 1533170,1991$. 